\title{
EFECTOS DE LA DIURESIS ACUOSA SOBRE EL pH URINARIO DE LOS PACIENTES LITIÁSICOS RECIDIVANTES
}

\author{
R.A. MEDINA LÓPEZ*, J. RIBAS SERNA**, F. GARCÍA MATILLA* \\ *Servicio de Urología. H.U. Virgen del Rocío. Sevilla. **Departamento de Fisiología. Facultad de Medicina. \\ Universidad de Sevilla.
}

Actas Urol Esp. 27 (5): 361-369, 2003

\section{RESUMEN}

"EFECTOS DE LA DIURESIS ACUOSA SOBRE EL pH URINARIO DE LOS PACIENTES LITIÁSICOS RECIDIVANTES" INTRODUCCIÓN: En individuos sanos se ha observado que el aumento de flujo urinario inducido por una sobrecarga acuosa puede ser suficiente para desviar el $\mathrm{pH}$ urinario a valores de seguridad para la profilaxis de la litogénesis. El propósito del presente trabajo es conocer los efectos de la diuresis acuosa sobre el pH urinario en pacientes con enfermedad litiásica recidivante.

MATERIAL Y MÉTODO: Se incluyeron 26 pacientes. A todos se les determinó el pH urinario, dos días consecutivos, a las 8.30 y las 12.30 horas, en condiciones normales ( $1^{\mathrm{er}}$ día) y sometiéndolos a una carga acuosa correspondiente al $1,5 \%$ de su peso corporal ( $2^{\circ}$ día).

RESULTADOS: La modificación de la media del $\mathrm{pH}$ inducida el $1^{\mathrm{er}}$ día por el ritmo circadiano se situó en una media de 0,13 unidades de $\mathrm{pH}$, valor sin significación estadística ( $\mathrm{p}>0,05)$, mientras que el $2^{\circ}$ día fue de 0,42 , estadísticamente significativo $(\mathrm{p}<0,01)$. La tendencia general tras la sobrecarga acuosa fue un incremento positivo del $\mathrm{pH}$ en aquellos pacientes cuya orina precarga poseía un $\mathrm{pH}$ inferior a 6,1 , mientras que los sujetos con $\mathrm{pH}$ precarga superior a 6,48 sufrieron incrementos negativos.

CONCLUSIONES: El aumento de la diuresis inducida por la carga acuosa fue suficiente para producir un aumento del pH urinario cuando en situación basal era inferior a 6,1 o una disminución si en condiciones basales era superior a 6,48, desviando en ambos casos el $\mathrm{pH}$ hacia valores comprendidos entre 6-6,5, nivel de seguridad para la profilaxis de la litiasis. Se ha observado mayor efecto de la carga acuosa cuanto más alejado del rango 6,1-6,48 estuvo el valor de $\mathrm{pH}$ urinario basal.

PALABRAS CLAVE: pH urinario. Diuresis acuosa. Sobrecarga acuosa. Litiasis urinaria.

\section{ABSTRACT}

"EFFECTS OF WATER DIURESIS ON THE URINARY pH OF PATIENTS UNDER RELAPSING LITHIASIS"

INTRODUCTION: It has been observed that in healthy individuals the increase in urinary flow induced by water overload may be enough to alter the urinary $\mathrm{Ph}$ to obtain values considered safe in the prophylaxis of lithogenesis. The aim of the present paper is to determine the effects of water diuresis on the urinary $\mathrm{Ph}$ of patients suffering from relapsing lithiasis.

MATERIAL AND METHOD: 26 patients were included in the present study. The urinary Ph of all patients was measured along two consecutive days, at 8.30 and again at 12.30 , under normal conditions (the first day) and once they had received a water load equivalent to $1.5 \%$ of their weight (the second day).

RESULTS: The altered mean value of the $\mathrm{Ph}$ induced on the first day by the circadian rhythm showed a mean of 0.13 units of $\mathrm{Ph}$, which lacks statistical significance $(\mathrm{p}>0.05)$. However, on the second day we obtained a value of 0.42 , which is statistically significant $(\mathrm{p}<0.01)$. The normal tendency after the water overload was towards a positive increase of the $\mathrm{Ph}$ in those patients whose urine showed a preload $\mathrm{Ph}$ value $<6.1$, whereas those individuals with a preload $\mathrm{Ph}$ value $>6.48$ suffered negative increases.

CONCLUSIONS: The increase of diuresis induced by water load proved enough to provoke an increase of urinary $\mathrm{Ph}$ when its baseline value is $<6.1$, or either a decrease when its baseline value is $>6.48$. In both cases, Ph values ranged between 6-6.5 which are considered safe values in the prophylaxis of lithogenesis. The more separated the baseline urinary $\mathrm{Ph}$ is from the 6.1-6.48 range, the greater the effect of the water load. 
$\mathrm{C}$ omo es conocido, en el desarrollo de la litiasis urinaria se encuentran implicados diversos factores, entre los que destacan: el aumento absoluto (por el aumento de la cantidad de cristaloides) o relativo (por un bajo volumen urinario) de la concentración de cristaloides en la orina, las desviaciones del pH urinario, la disminución de la concentración de inhibidores en la orina y la presencia de lugares de nucleación.

Entre estos factores destacan, por su mayor importancia en la litogénesis, el volumen de orina $\mathrm{y}$ el $\mathrm{pH}$ urinario.

Centrándonos en el primero, el aumento del volumen urinario produce una disminución en la concentración del soluto, aumenta la constante dieléctrica, facilita el arrastre de los núcleos cristalinos, produce cambios en los inhibidores de la cristalización y reduce la concentración de microorganismos. Por todo ello su influencia en el desarrollo de una litiasis es fundamental.

Corroborando estos hechos, Pak y cols. observan como el aumento del volumen urinario por encima de 2 litros al día produce una disminución en la saturación urinaria de oxalato cálcico, brushita y urato monosódico, así como la inhibición de la nucleación espontánea. Por ello, proponen para el tratamiento de la litiasis una adecuada hidratación y una dieta apropiada ${ }^{1}$.

Por otro lado, Fraioli en 1989 comunica como el aumento de la ingesta de líquidos conlleva un aumento de la diuresis, con disminución de la concentración de sales y de micro-organismos en orina, produce cambios en los mecanismos de concentración renal, influye en la inhibición de la cristalización, y provoca cambios en el pH urinario, además del efecto mecánico de arrastre ${ }^{2}$.

Cauderella en 1998 publica que los formadores de litiasis poseen mayor riesgo de litogénesis si su diuresis es inferior a 1 litro diario ${ }^{3}$. Por el contrario, el aumento de la diuresis produce una disminución del tiempo de contacto con la superficie absortiva de la nefrona ${ }^{4}$, disminuye los promotores de la litogénesis ${ }^{1}$, y aumenta la excreción de citrato ${ }^{5}$ y la actividad de la proteína de Tamm-Horsfall ${ }^{6}$; por todo ello disminuye el riesgo de la formación de cristales.

Por el contrario Coe y Laerum no reconocen relación entre la hidratación y la dieta con las recidivas litiásicas ${ }^{7,8}$.
Marsh y Stuart ven como la respuesta a una sobrecarga estándar de agua es mayor por la mañana ${ }^{9,10}$.

En cuanto a la influencia del tipo de agua ingerida en el desarrollo de litiasis, se pensaba que existía una relación directa entre el consumo de aguas duras y la producción de cálculos urinarios. Sin embargo, existen estudios que evidencian una correlación negativa entre la dureza del agua y el riesgo de litogénesis ${ }^{11,12}$, así como una mayor incidencia de cálculos en zonas de aguas blandas ${ }^{11,13}$.

Vallejo en 1992 en un estudio realizado en España sobre 29 tipos de agua, publica como primera conclusión que es más importante la cantidad de agua ingerida que su composición ${ }^{14}$. Este hecho fue igualmente publicado por Cauderella ${ }^{3}$.

El otro factor determinante en la litogénesis es el $\mathrm{pH}$ urinario. Las diferentes sales precipitan a diferentes $\mathrm{pH}$, de esta forma el ácido úrico fácilmente precipita a $\mathrm{pH}$ urinario inferior a 5,5, mientras que lo hace con dificultad a $\mathrm{pH}$ igual o mayor a 6. El fosfato precipita con mayor facilidad a pH mayor de 6,5, y lo hace difícilmente a $\mathrm{pH}$ inferior a 6. Las sales de oxalato y fosfato precipitan primordialmente a $\mathrm{pH}$ mayor de 6,4 y las de oxalato cálcico en $\mathrm{pH}$ inferiores a $6^{14-24}$. Vemos como el pH de mayor seguridad para la litogénesis, en general, se sitúa en el intervalo 6-6,5.

En el proceso de precipitación es importante reseñar en todo momento a que temperatura y $\mathrm{pH}$ se desarrolla, ya que un cambio en cualquiera de los dos factores modifica sustancialmente la cantidad de soluto que puede mantenerse es suspensión ${ }^{25-27}$. En el ser humano la temperatura es una variable bien mantenida. Por tanto las variaciones en décimas de unidades de $\mathrm{pH}$ producen cambios importantes en el estado de saturación de las sales.

Wilson y cols. estudiando el crecimiento de los cristales de hidroxiapatita, observan como al aumentar el $\mathrm{pH}$ aumenta la actividad inhibitoria del pirofosfato y del citrato $^{28}$.

Thode en $1993^{29}$ y posteriormente Langley en $1997^{30}$ describen como la actividad iónica del calcio desciende conforme el $\mathrm{pH}$ urinario aumenta, manteniendo una relación bifásica en torno a un pH crítico. 
Por otro lado, al analizar la influencia del pH urinario en la litogénesis, debemos tener en cuenta que éste se encuentra sometido a variaciones cíclicas a lo largo del día. De esta forma Eliot en 1959 define 4 patrones de ritmo circadiano del $\mathrm{pH}$ urinario, siendo el más típico aquel que presenta un $\mathrm{pH}$ urinario bajo por la mañana, se eleva durante la "marea alcalina" y mantiene un pH bajo a última hora del día ${ }^{31}$. Posteriormente, en 1993 Murayama describe 3 patrones de variaciones rítmicas del pH urinario a lo largo de las 24 horas, cada una dividida en varios subtipos; en líneas generales, los pacientes que poseen el tipo $1 \mathrm{de}$ ritmo mantienen durante todo el día su $\mathrm{pH}$ urinario por debajo de 6 , el tipo 2 presenta fluctuaciones en torno a 6 a lo largo de las 24 horas, y el tipo 3 mantiene el pH siempre por encima de 6. Este autor no encontró diferencias entre individuos sanos y formadores de litiasis en relación a los tipos 1 y 2 , mientras que si existían en relación al tipo $3^{32}$.

Por último se ha descrito en condiciones de laboratorio y en individuos sanos relación entre los dos factores analizados, el volumen urinario y el pH. De esta forma se ha visto como un flujo urinario bajo, se corresponde generalmente con una orina concentrada y un $\mathrm{pH}$ bajo, mientras que un flujo alto lo hace generalmente con una orina diluida y un $\mathrm{pH}$ alto.

Engelton en 1946 y Barclay en 1947 publican como durante la diuresis acuosa el $\mathrm{pH}$ urinario se aproxima a 6 , independientemente del $\mathrm{pH}$ previo $^{33,34}$. Reid y Hills en 1965 comprueban que el $\mathrm{pH}$ urinario es función del flujo de orina ${ }^{35}$. Tannen en 1969 llega a cuantificar esta relación, comprobando un incremento de 0,35 unidades de $\mathrm{pH}$ por cada $7,2 \mathrm{ml} / \mathrm{min}$ de incremento de flujo ${ }^{36}$; y García Matilla describe en sujetos sanos que el aumento de flujo urinario induce un incremento medio de 0,57 unidades de $\mathrm{pH}^{37,38}$.

El objetivo principal de este trabajo es determinar los cambios del $\mathrm{pH}$ urinario de sujetos litiásicos recidivantes producidos por una sobrecarga oral de agua. En definitiva demostrar que no sólo la diuresis acuosa y el pH urinario por sî mismos son factores en la litogénesis, si no que la diuresis acuosa a través de su efecto sobre el $\mathrm{pH}$ de la orina es capaz igualmente de influir sobre el proceso litogénico.

\section{MATERIAL Y MÉTODO}

Como material hemos empleado a la población litiásica recidivante del Área Hospitalaria Virgen del Rocío de Sevilla.

Se han utilizado como criterios de inclusión ser mayor de 18 años, haber sufrido al menos dos episodios litiásicos en los últimos 2 años, diagnosticados por su urólogo, poseer una función renal normal y aceptar por escrito la inclusión en el estudio una vez informado.

Como criterios de exclusión el estar en tratamiento médico para su litiasis y tomar fármacos que alteren el $\mathrm{pH}$ urinario.

La selección de los sujetos se ha realizado teniendo en cuenta también que la actividad física y psíquica fueran semejantes en todos ellos, con un nivel de responsabilidad hacia el cumplimiento de los requerimientos para la recogida de muestras que permitiera asegurar condiciones homogéneas.

El tamaño de la muestra se calculó para obtener un error alfa de 0,001 con una potencia estadística del 90\%, precisándose para ello a 22 pacientes, que se suplementaron con 4 como proporción de seguridad, sumando un total de 26 individuos, con una edad media de 44 años (rango entre 31 y 66) y una distribución por sexo de 13 hombres (50\%) y 13 mujeres (50\%).

Las variables estudiadas, todas ellas dependientes, fueron en orina la diuresis de 4 horas, el flujo, el pH y osmolaridad; y en sangre la osmolaridad.

Todos los pacientes durante las 8 horas previas a iniciar el estudio, que coincidieron con la noche, estuvieron sometidos a dieta absoluta.

A todos se le recogió la diuresis durante un periodo de tiempo idéntico, 240 minutos, entre las 8:30 y las 12:30 h de dos días consecutivos e igualmente se tomó una muestra de orina reciente a ambas horas de ambos días.

El pH urinario se determinó inmediatamente tras obtener la muestra de orina reciente a las 8:30 y a las 12:30 de ambos días.

Durante el primer día (día 1) los pacientes realizaron su actividad normal durante el tiempo que duró la prueba, de esta forma los cambios observados entre la medición del $\mathrm{pH}$ a primera y última hora deben ser atribuidos a los cambios motivados por el ritmo circadiano. 
El segundo día (día 2) se actuó de la misma forma, salvo que durante las 8:30 y las 9:30 h los pacientes ingirieron una cantidad de agua correspondiente al $1,5 \%$ de su peso. De esta forma los cambios encontrados entre ambas determinaciones deben ser atribuidos a la única variación existente, la sobrecarga acuosa.

Ambos días también se determinaron el flujo urinario y la osmolaridad en orina y sangre.

Todos los datos fueron incluidos en una base de datos creada para este fin, realizándose una evaluación estadística básica mediante el programa Rsigma. Como test estadístico para evaluar los diferentes resultados bioquímicos se ha utilizado la T de Student, y se ha calculado el coeficiente de correlación entre las diferentes variables.

\section{RESULTADOS}

El pH medio alcanzado a primera y última hora de ambos día fueron los expresados en la Tabla I.

La diferencia entre el $\mathrm{pH}$ a las 8:30 y 12:30 h del primer día (condiciones normales) no tuvo significación estadística, mientras que si la hubo entre los pH de primera y última hora del segundo día con la sobrecarga acuosa. De la misma manera la diferencia fue significativa entre el $\mathrm{pH}$ de las 12:30 de ambos días ( $\mathrm{p}<0,01$ ).

$\mathrm{El}$ día 1 en condiciones normales, por el ritmo circadiano se obtuvo un incremento medio del $\mathrm{pH}$ de 0,13 U (Fig. 1), mientras que el día 2 con la sobrecarga este incremento fue de 0,42 $\mathrm{U}$ de $\mathrm{pH}$ (Fig. 2), presentando una diferencia estadísticamente significativa $(\mathrm{p}<0,01)$.

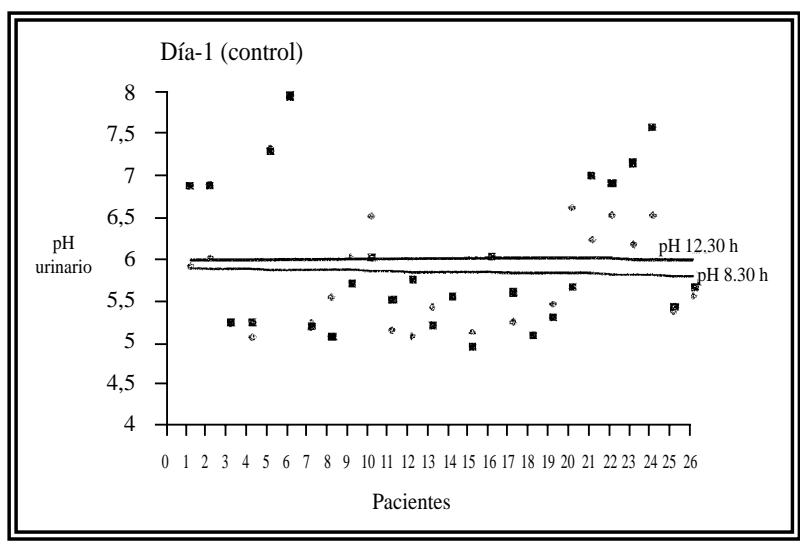

FIGURA 1. Curvas de regresión de las medidas del pH a las 8:30 y a las 12:30 h. del primer dia (control). La diferencia sufrida no tiene significación estadistica $(p>0,05)$.

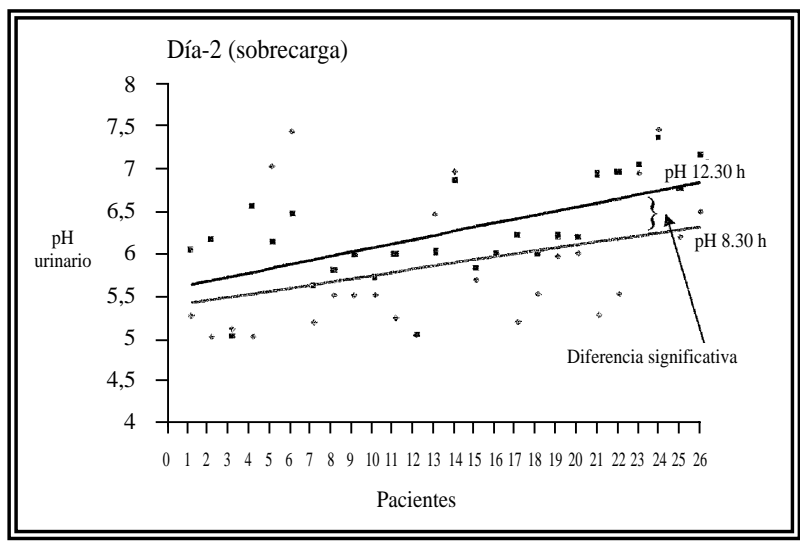

FIGURA 2. Curvas de regresión de las medidas del pH a las 8:30 y a las 12:30 h. del segundo día (sobrecarga acuosa). La diferencia sufrida tiene significación estadistica $(p<0,05)$.

Las variaciones del $\mathrm{pH}$ de cada individuo cada día entre primera y última hora de la mañana podemos apreciarlas en la Figura 3 (en condiciones

\section{TABLA I}

VALORES DEL pH MEDIO, DESVIACIÓN TÍPICA, pH MÍNIMO Y MÁXIMO Y RANGO

\begin{tabular}{||l|c|c|c|c|c||}
\hline & PH medio & $\begin{array}{c}\text { Desviación } \\
\text { Típica }\end{array}$ & Mínimo & Máximo & Rango \\
\hline $\begin{array}{l}\text { Día } 1 \\
8: 30 \mathrm{~h}\end{array}$ & 5,8 & 0,77 & 5 & 8 & 3 \\
\hline $\begin{array}{l}\text { Día } 1 \\
12: 30 \mathrm{~h}\end{array}$ & 5,93 & 0,90 & 4,87 & 7,5 & 2,5 \\
\hline $\begin{array}{l}\text { Día } 2 \\
8: 30 \mathrm{~h}\end{array}$ & 5,86 & 0,79 & 5 & 5,4 & 2,4 \\
\hline $\begin{array}{l}\text { Día } 2 \\
12: 30 \mathrm{~h}\end{array}$ & 6,28 & 0,56 & 5 & 2 & 3 \\
\hline
\end{tabular}




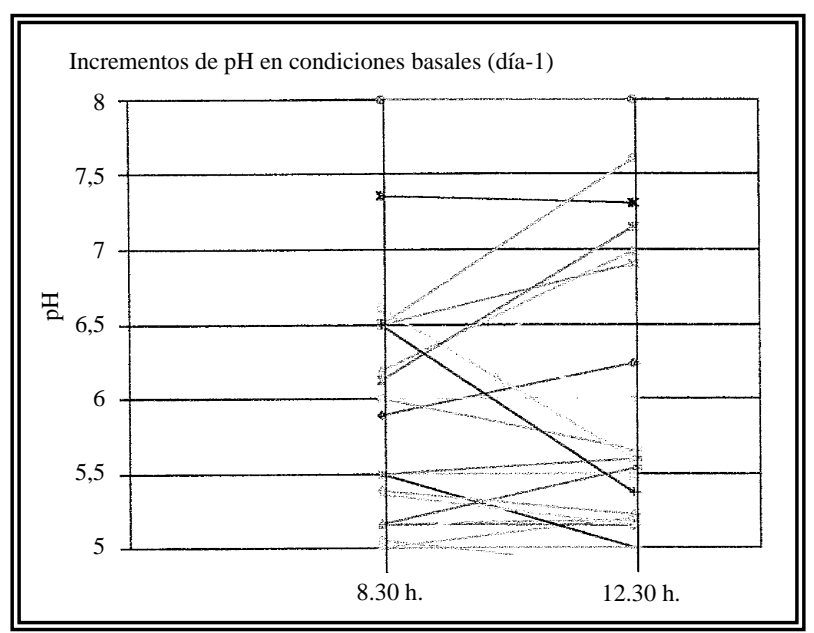

FIGURA 3. Valor del pH de cada paciente a las 8.30 h. y a las $12.30 \mathrm{~h}$. del primer dia.

normales) y Figura 4 (con la sobrecarga a acuosa), observando como con la sobrecarga acuosa los valores de $\mathrm{pH}$ tienden a agruparse en torno a una media de 6,28.

La tendencia general consistió en el descenso de los incrementos de $\mathrm{pH}$ post-carga conforme mayores fueron los valores de $\mathrm{pH}$ precarga. Pero analizando esta tendencia se puede observar como los pacientes cuyos valores de $\mathrm{pH}$ precarga eran inferiores a 6,1 presentaron incrementos positivos y cuanto más ácida fue la orina precarga mayor fue el efecto de la carga acuosa sobre su $\mathrm{pH}$. Por el contrario la mayoría de los incrementos negativos lo sufrieron aquellos individuos cuyos valores de $\mathrm{pH}$ iniciales eran superiores a 6,48 (Fig. 5).

Se evidenció de esta forma como el primer día, debido a las variaciones fisiológicas, sólo el $11,54 \%$ de los pacientes estudiados poseían a última hora de la mañana un $\mathrm{pH}$ urinario entre 6 y 6,5 , rango de menor poder litogénico. Sin embargo, el segundo día tras la sobrecarga acuosa, el $57,69 \%$ de los pacientes lograron situarse en este rango, y el resto experimentaron cambios que se aproximaban a estos valores.

El volumen medio de orina obtenido durante las 4 horas de recogida durante el día 1 (condiciones normales) fue de 209,46 ml (Desviación Típica (D.T.) 104,71), mientras que durante el segundo, con la sobrecarga acuosa, se elevó a 509,81 ml (D.T. 308,59). A pesar de que los aumentos fueron diferentes entre los diferentes

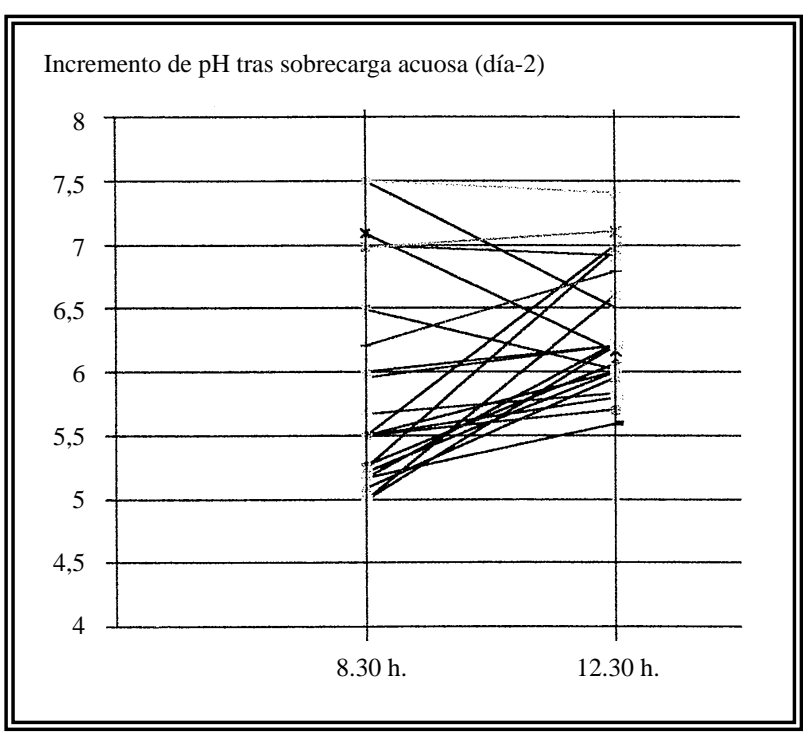

FIGURA 4. Valores del pH urinario de cada paciente a las 8.30 y a las $12.30 \mathrm{~h}$ del segundo dia.

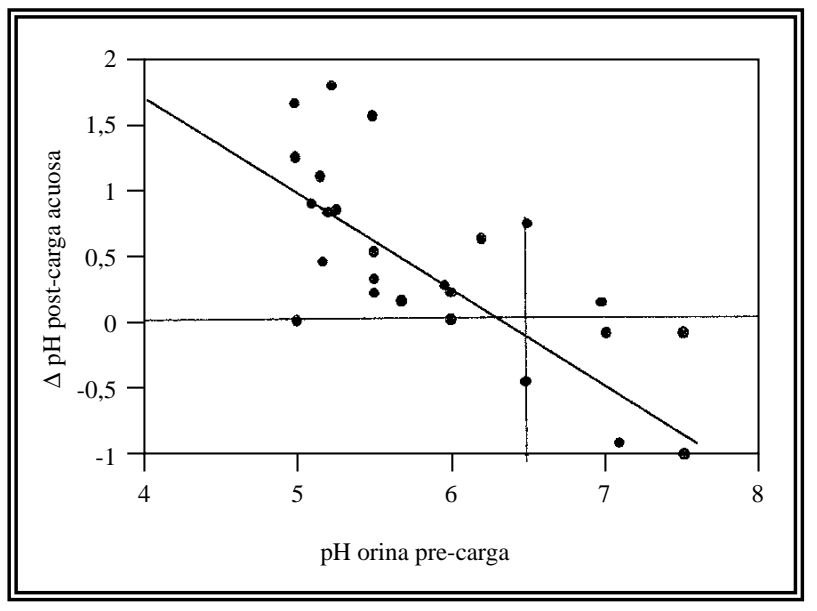

FIGURA 5. Dependencia de los incrementos de pH postcarga acuosa respecto a los pH de la orina en condiciones basales (pre-carga).

individuos, los valores medios mostraron diferencias estadísticamente significativas, con incremento medio de $381,35 \mathrm{ml}$ (D.T. 333,94).

La media del flujo urinario en el primer día fue de $0,83 \mathrm{ml} / \mathrm{min}$ (D.T. 0,43), mientras que el segundo día fue de $2,50 \mathrm{ml} / \mathrm{min}$ (D.T. 1,34). La diferencia entre estos valores es estadísticamente significativa $(p<0,001)$, con incremento medio experimentado de $1,62 \mathrm{ml} / \mathrm{min}$ (D.T. 1,44).

Cuando se relacionaron los cambios del $\mathrm{pH}$ con el flujo de orina se puso de manifiesto que a medida que aumentó el flujo por efecto de la carga acuosa, lo hizo también el pH ( $\mathrm{R}=0$,33555) (Fig. 6). 


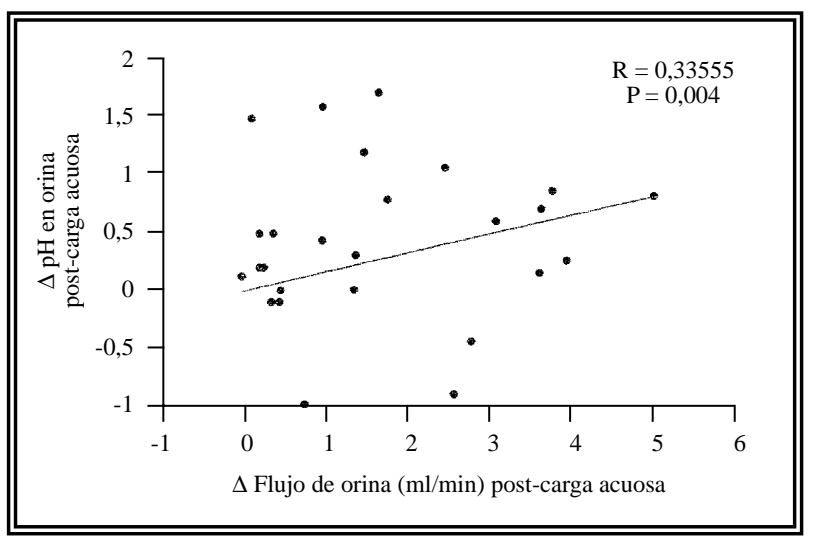

FIGURA 6. Correlación entre los incrementos de flujo urinario y de pH tras la administración de una sobrecarga acuosa.

Se ha podido establecer una relación positiva entre los aumentos del flujo urinario y los incrementos del $\mathrm{pH}$.

La osmolaridad urinaria varió de 715,88 mOsm/1 (D.T. 232,08) el primer día a 385,38 (D.T. 214,02) el segundo, presentando una diferencia significativa $(\mathrm{p}<0,001)$.

Los cambios en la diuresis con la sobrecarga acuosa se corresponden con decrementos de la osmolaridad urinaria, aunque la relación entre ambas variables no siguió un curso lineal, ajustándose a una función polinómica (Fig. 7).

La osmolaridad plasmática, al igual que la urinaria, experimentó cambios significativos, desde una media de 290,81 (D.T. 7,45) el primer día a 287,38 (D.T. 6,66) el segundo. Por ello, podemos decir que el volumen de agua utilizado (equiva-

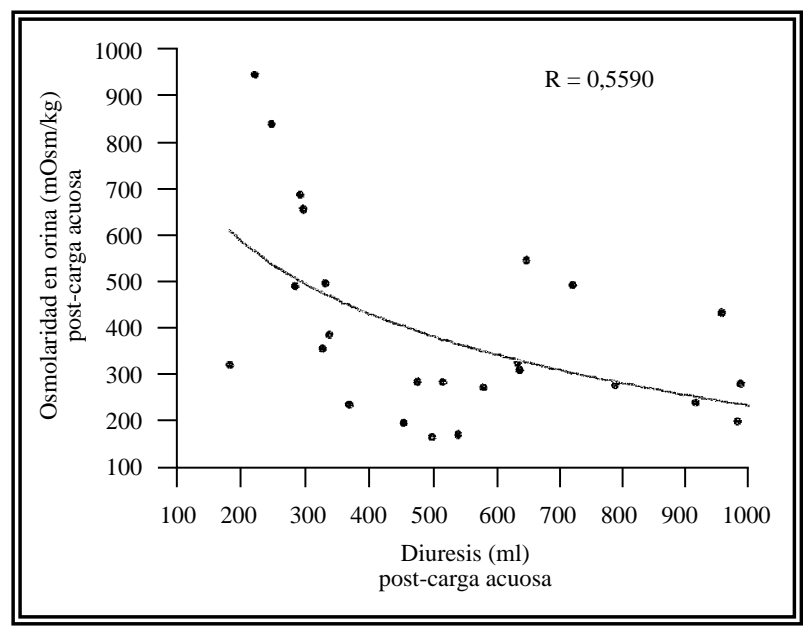

FIGURA 7. Relación entre la diuresis post-carga acuosa (ml) y la osmolaridad urinaria alcanzada. lente al $1,5 \%$ del peso corporal del paciente) es suficiente para inducir la expansión del volumen extracelular.

\section{DISCUSIÓN}

La orina no se comporta como un disolvente común. La formación y crecimiento de cristales para producir cálculos depende de factores físicoquímicos complejos, influyendo de manera importante el volumen y el pH urinario. Por este motivo, los estados de hiposaturación, saturación y sobresaturación de las disoluciones acuosas no son comparables a los de la orina ${ }^{25,39-41}$.

Los efectos beneficiosos de la diuresis acuosa sobre la litogénesis se justifican por la disminución de la concentración relativa de los componentes litiásicos, facilitar el arrastre de núcleos cristalinos, producir cambios en los inhibidores de la cristalización, reducir la concentración de micro-organismos y por los cambios producidos en el $\mathrm{pH}$ urinario ${ }^{14,42-47}$.

También es conocido que la concentración de las diferentes sales varía en relación al $\mathrm{pH}$ urinario $^{15-24,48}$. En este sentido, Hallson y Rose ${ }^{22,23}$ observaron que las concentraciones mínimas de cristales de oxalato cálcico se consiguen con valores de pH entre 6,0 y 6,4 , coincidiendo con Robertson ${ }^{49,50}$.

Para el objetivo de disminuir el riesgo de precipitación de sales urinarias promotoras de cálculos no hacen falta efectos cuantitativos importantes sobre el $\mathrm{pH}$ urinario, ya que es suficiente con que aumente o disminuya en 0,2-0,4 unidades para que el cambio tenga mucha importancia en relación a la solubilidad de las sales urinarias, puesto que pueden ser suficientes para modificar sustancialmente el porcentaje de la forma ionizada y no ionizada, dadas las características de las curvas de ionización ${ }^{17,21,24,51-53}$.

De esta forma tenemos, por un lado, que los cambios en el pH urinario pueden alterar la solubilidad de las sales y por otro, las variaciones que en dicho $\mathrm{pH}$ puede producir el aumento del flujo urinario.

Son escasos los estudios sobre la relación del $\mathrm{pH}$ urinario relacionado con el flujo de la orina y los mecanismos que pueden explicar esta tendencia. En este trabajo hemos pretendido estudiar dicha relación. 
Como se expuso en la introducción, el rango de $\mathrm{pH}$ entre 6 y 6,5 es el de menor poder litogénico, debido fundamentalmente a la disminución en la concentración de cristales en orina, disminuir la nucleación espontánea y aumentar la actividad inhibitoria de pirofosfato y citrato.

Con el ritmo circadiano, es decir, con las variaciones cíclicas fisiológicas del $\mathrm{pH}$ urinario, sólo el 11,45\% de los individuos del presente estudio alcanzaron este rango, mientras que con la diuresis acuosa lo lograron el 57,69\% de ellos.

Era conocido como en sujetos sanos la sobrecarga acuosa produce un incremento medio de 0,57 unidades de $\mathrm{pH}$, alcanzando un $\mathrm{pH}$ urinario medio de 6,22 ${ }^{37,38}$. En el presente trabajo hemos visto que en los pacientes litiásicos recidivantes este incremento es de 0,42 unidades de $\mathrm{pH}$, alcanzando un $\mathrm{pH}$ urinario medio de 6,28. Es decir la diuresis acuosa logra situar el pH de estos pacientes en valores de seguridad para la litogénesis.

En relación a estos efectos de la diuresis acuosa sobre el $\mathrm{pH}$ de la orina, objetivo central del presente trabajo, se sabe que tanto en el humano como en los animales, generalmente y en condiciones normales, las diuresis de alto volumen coinciden con $\mathrm{pH}$ de orina alto, alcalino, mientras que las diuresis de bajo volumen coinciden con pH bajo, ácido. Los mecanismos reguladores de la secreción tubular de $\mathrm{H}^{+}$están influidos por el pH del líquido tubular. Ello explicaría que la relación entre el volumen y el $\mathrm{pH}$ de la orina en determinadas condiciones pudiera ser distinta, dependiendo del $\mathrm{pH}$ previo.

Igualmente era conocido en condiciones in vitro como a flujo urinario máximo si el $\mathrm{pH}$ de la orina es mayor o igual a 6,6 , el pH varía inversamente al flujo y si lo es menor a 4,3 lo hace de manera directa ${ }^{35}$. Es decir, si el pH urinario es ácido el aumento de flujo urinario induce una tendencia a la alcalinización y si por el contrario el pH urinario es básico este mismo aumento de flujo produce una tendencia a la acidificación. Con ello, la relación entre el volumen y el pH urinario puede ser directa o inversa dependiendo del $\mathrm{pH}$ urinario de partida.

En nuestro estudio se ha demostrado que si el $\mathrm{pH}$ urinario de partida es menor a 6,1 el aumento del flujo urinario produce una elevación del mismo, mientras que si este $\mathrm{pH}$ basal es superior a 6,48 este aumento del flujo conlleva su descenso. Por todo esto podríamos hablar de un efecto modulador de la diuresis acuosa sobre el $\mathrm{pH}$ urinario.

En cuanto a los mecanismos que pueden explicar este fenómeno, según los trabajos publicados por Bichara en 1984, serían los siguientes ${ }^{54}$ :

1. Si el pH urinario inicial es ácido (menor de 6) la expansión del volumen conllevaría una disminución de la secreción de hidrogeniones por la inhibición de transporte sodio y agua, así como la disminución de la reabsorción de bicarbonato. Esto implicaría un ascenso del $\mathrm{pH}$ urinario.

2. Por el contrario si el pH urinario inicial es alcalino (por encima de 6,5), la expansión de volumen produciría una elevación de la secreción de hidrogeniones por la activación del antiporte sodio-hidrogeniones e hidrogeniones-ATPasa, así como la reabsorción pasiva de bicarbonato. Esto implicaría un descenso del $\mathrm{pH}$ urinario.

Todo ello corrobora que una adecuada ingestión de agua puede desviar el pH urinario hacia la zona de seguridad para la prevención de litiasis, descendiéndolo en el caso de $\mathrm{pH}$ urinarios alcalinos o elevándolo en el caso contrario, por ello podemos hablar de un efecto modulador de la carga acuosa sobre el pH urinario.

Los cambios expresados en el $\mathrm{pH}$ de la orina a consecuencia de la diuresis acuosa pueden ser explicados por efectos debidos a modificaciones del volumen del líquido extracelular, del filtrado glomerular, del pH luminal previo, cambios en la velocidad del flujo luminal de la nefrona, del $\mathrm{pH}$ peritubular y $\mathrm{PCO}_{2}$, PTH y $\mathrm{K}^{+}$. Los mecanismos que intervienen actúan de tal manera que, cuando el $\mathrm{pH}$ inicialmente es alto son capaces de causar secreción de $\mathrm{H}^{+}$y reabsorción de $\mathrm{HCO}_{3}{ }^{-}$que tienen como consecuencia final la disminución del $\mathrm{pH}$ hasta un valor límite. Por el contrario, cuando el pH inicial es ácido, los mismos mecanismos giran en su forma de actuar de tal manera que causan aumento del $\mathrm{pH}$ hasta un valor límite. Según los datos obtenidos en este estudio se sugiere que el valor límite está situado entre pH 6,1-6,48.

Nuestros resultados muestran cambios en las variables sanguíneas compatibles con una expansión del volumen extracelular. El descenso significativo de la osmolaridad experimentada entre el primer día del estudio (sin sobrecarga 
acuosa) y el segundo (con sobrecarga), asegura que el volumen de agua elegido para la carga acuosa fue suficiente para inducir una expansión notable del volumen en el espacio extracelular, incidiendo, por tanto, en aquellos mecanismos de reabsorción y secreción tendentes a modular el pH de la orina hacia la zona óptima de disolución de solutos.

\section{CONCLUSIONES}

- En la población de litiásicos recidivantes estudiados, una sobrecarga oral de agua equivalente a un $1,5 \%$ del peso corporal tuvo un efecto expansor sobre el volumen del compartimento líquido extracelular, lo que queda demostrado por los cambios hallados en la osmolaridad plasmática.

- La carga acuosa utilizada fue suficiente para producir un aumento de la diuresis a valores capaces de activar mecanismos reguladores de $\mathrm{pH}$ de la orina. El hecho de que la carga acuosa se ingiriese en un plazo de tiempo relativamente corto, indujo un aumento del flujo de orina compatible con cambios en los gradientes de concentración transtubular aptos para modificar el pH de la orina final.

- El aumento del flujo de orina inducido por la carga acuosa fue suficiente para producir un aumento medio de 0,42 unidades de pH. Esto desvió el pH del grueso de los individuos estudiados hacia valores de seguridad para la profilaxis de la litiasis recidivante.

- Se ha constatado un mayor efecto de la carga acuosa cuanto más alejado del rango 6,16,48 estuvo el valor de $\mathrm{pH}$ de la orina pre-carga.

- El aumento de la ingestión de agua es eficaz para la profilaxis de la litiasis recidivante debido a los efectos de modulación del pH urinario, el cual es conducido por la diuresis acuosa a valores comprendidos entre pH 6 a 6,5, que son, precisamente, los que implican menor porcentaje de litiasis urinaria.

- La ausencia de las respuestas descritas por algunos de los pacientes sometidos a la carga oral de agua sugiere que existen defectos en los mecanismos renales de acidificación de la orina.

- El aumento de la diuresis tras la sobrecarga hídrica conlleva una disminución de la osmolaridad urinaria. La relación entre ambas variable se ajusta a una función polinómica.

\section{REFERENCIAS}

1. PAK CYC, SAKHAEE K, CROWTHER C, BRINKLEY L.: Evidence justifying a high fluid intake in treatment of nephrolithiasis. Ann Intern Med 1980; 93: 36.

2. FRAIOLI A, NOCCHI S, MENNUNI G, MAMMUCARI S, FONTANA M, MESSINA B.: Hydrological therapy of renal lithiasis. Clin Ter 1989; 131 (5): 321-32-30.

3. CAUDARELLA R, RIZZOLI E, BUFFA A, BOTTURA A, STEFONI S.: Comparative study of the influence of e types of mineral water in patients with idiopathic calcium lithiasis. J Urol 1998; 159: 658-663.

4. FINLAYSON B.: Symposium on renal lithiasis. Renal lithiasis in review. Urol Clin North Am 1974; 1: 181.

5. HESS B, MICHEL R, TAKKINEN R, ACKERMANN D, JAGER P.: Risk factors for low urinary citrate in calcium nephrolithiasis: low vegetables fiber intake and low urine volume to be added on the list. Nephrol Dial Transplant 1994; 9: 642 .

6. HESS B, ZIPPERLE L, JAEGERE P.: Citrate and calcium effects on Tamm. Horsfall glycoprotein as a modifier of calcium oxalate crystal aggregation. Amer $J$ Phsyol 1993; 265: F784.

7. COE FL.: Treated and untreated recurrent calcium nephrolithiasis in patients with idiopathic hypercalciuria, hyperuricosuria, or no metabolic disorder. Ann Intern Med 1977: 87: 404-410.

8. LAERUM E.: Recurrent urolithiasis: a general-practice study of risk factors and clinical consequences. Scand J Urol Nephrol 1983; 18: 67-70.

9. MARSH DJ.: Renal physiology. Raven Press. New York, 1983.

10. STUART RO II, HILL K, POINDEXTER J et al.: Seasonal variations in urinary risk factors among patients with nephrolithiasis. J Lithotr Stone Dis 1991; 3: 18.

11. SHUSTER J, FINLAYSON B, SCHEAFFER R et al.: Water hardbess and urinary stone disease. $J$ Urol 1982; 128: 422.

12. ROSE GA, WESTBURY EJ.: The influence of calcium content of water, intake of vegetables and fruit and other food factors upon the incidence of renal calculi. Urol Res 1975; 3: 61.

13. CHURCHILL D, BRYANT D, FODOR G, GAULT MH.: Drinking water hardness and urolithiasis. Ann Int Med 1978; 88: 513.

14. VALLEJO HERRADOR J, GALMES BELMONTE I, BURGOS REVILLA FJ y cols.: Valor real del aporte de líquidos y sus cualidades, en la población litiásica. Actas Urol Esp 1992; 16: 233-239.

15. GRASES F, CONTE A, COLL R, GENESTAR C.: The role of hyperoxaluria in the formation of calcium oxalate urinary calculi, and its association with other biochemical measurements. Scand J Urol Nephrol 1990; 24: 211-213.

16. PAK CYC.: Pathophysiology of calcium nephrolithiasis. In: The Kidney: physiology and pathophysiology, Seldin and Giebisch (ed). Raven Press. New York 1985; 2: 1365-1379.

17. ABRAHAM PA, SMITH CL.: Medical evaluation and management of calcium nephrolithiasis. Med Clin North Am 1984; 68: 281-299.

18. CIFUENTES DELATTE L.: Treinta años de tratamiento alcalinizante. Actas Urol Esp 1990; 2: 85-88.

19. CIFUENTES DELATTE L.: El tratamiento alcalinizante como preventivo de la litiasis oxalocálcica recidivante. Arch Esp Urol 1991; 8: 903-910. 
20. CONTE A, GENESTAR C, GRASES F.: Relation between calcium oxalate hydrate form found in renal calculi and some urinary parameters. Urol Int 1990; 45: 2527.

21. DAUDON M, JUNGERS P.: Patogenia de la litiasis urinaria. En Litiasis urinaria. Editado por Caralps A, Rapado A, Saladié JM y Pérez Castro E. Barcelona (España), Doyma ed. 1991: 3-11.

22. HALLSON PC, ROSE GA.: Risk factors for urinary calcium oxalate crystals as revealed by their specific enzymatic assay. Br J Urol 1989; 64: 451-457.

23. HALLSON PC, ROSE GA.: Measurement of calcium phosphate crystalluria: influence of $\mathrm{pH}$ and osmolality and invariable presence of oxalate. Br J Urol 1989; 64: 458-462.

24. MATEOS ANTÓN F.: Cálculos de ácido úrico. En: Litiasis urinaria. Editado por Caralps A, Rapado A, Saladié JM y Pérez Castro E. Barcelona (España), Doyma ed. 1991: 3-11.

25. BEGUN FP, FOLEY WD, PETERSON A, WHITE B.: Patient evaluation. Laboratory and imaging studies. Urol Clin North Am 1997; 24 (1): 97-116.

26. FINLAYSON B.: Symposium on renal lithiasis. Renal lithiasis in review. Urol Clin North Am 1974; 1: 181.

27. MANDEL NS, MANDEL GS.: Epitaxis between stoneforming crystals at the atomic level. En: Smith LH, Robertson WG, Finlayson B: Urolithiasis. Penum Press. New York 1981: 469.

28. WILSON JWL, WERNESS PG, SMITH LH.: Inhibidores del crecimiento de los cristales de hidroxiapatita: un estudio con composición constante. J Urol (Baltimore) 1985; 134/ 6: 1255-1258.

29. THODE J, HOLGERSEN RB, GERSTENBERG T.: Variability of the calcium ion activity with $\mathrm{pH}$ in stoneforming and non-stone forming urine. Scand J Clin Lab Invest Suppl, 1993; 214: 121-126.

30. LANGLEY SE, FRY CH.: The influence of pH on urinary ionized $\left[\mathrm{Ca}^{2+}\right]$ : differences between urinary tract stone formers and normal subjects. Br J Urol 1997; 79 (1): 814.

31. ELLIOT JS, SHARP RF, LEWIS L.: Urinary pH. J Urol 1959; 81: 339.

32. MURAYAMA T, TAGUCHI H.: The role of the diurnal variation of urinary $\mathrm{pH}$ in determinig stone composition. J Urol 1993; 150: 1437-1439.

33. EGGLETON MG.: Urine acidity in alcohol diuresis in man. J Physiol 1946; 104: 312-320.

34. BARCLAY JA, COOKE WT, KENNEY RA, NUTT ME.: The effects of water diuresis and exercise on the volume and composition of the urine. Amer J Physiol 1947; 148: $327-337$.

35. REID EL, HILLS AG.: Diffusion of carbon dioxide out of the distal nephron in man during antidiuresis. Clin Sci 1965; 28: 15-28.

36. TANNEN RL.: The relationship between urine $\mathrm{pH}$ and acid excretion. The influence of urine flow rate. $J \mathrm{Lab}$ Clin Med 1969; 74: 757-769.

37. GARCÍA MATILLA F, GARCÍA MONTES F, RIBAS SERNA J.: Relación entre la diuresis, $\mathrm{pH}$ de la orina y litogénesis. Actas Urol Esp 1999; 23 (3): 202-213.

38. GARCÍA MATILLA F, GARCÍA MONTES F, RIBAS SERNA J.: Diuresis acuosa y profilaxis de la nefrolitiasis recidivante. Actas Urol Esp 1999; 23 (4): 296-308.

39. BALAJI KC, MANI M.: Mechanism of stoneformation. Urol Clin North Am 1997; 24 (1): 1-11.
40. ASHBY RA, BYRNE JP, GYORY AZ.: Urine is saturated equilibrium and not metastable supersaturated solution: evidence from crystalluria and the general composition of calcium salt and uric acid calculi. Urol Res 1999; 27 (5): 297-305.

41. HESSE A, HEIMBACH D.: Causes of phosphate stone formation and the importance of metaphylaxis by urinary acidification: a review. World J Urol 1999; 17 (5): 308-315.

42. ANTÚNEZ F, PEDRAJAS A, LANCINA JA.: Epidemiología de la litiasis urinaria. En Ponencia LV Congreso Nacional Asoc Esp de Urol: Criterios clínicos y tratamiento actual de la litiasis urinaria (Vigo), ENE ed. 1990: 13-20.

43. TORRES RAMÍREZ C, NAVARRO SÁNCHEZ-ORTIZ A, ZULUAGA GÓMEZ A.: Influencias estacionales de la edad, sexo y composición del agua debida sobre los cólicos nefríticos. Arch Esp Urol 1981; 34: 273.

44. DEGANELLO S, COE FL.: Epitaxy between uric acid and whewellite. Experimental verification. $N$ Jb Miner Mh H-6 1983: 270-276.

45. KHATCHADOURIAN J, PREMINGER GM, WHITSON PA et al.: Clinical and biochemical presentation of gouty diathesis: comparison of uric acid versus pure calcium stone formation. J Urol 1995; 154: 1665.

46. ZULUAGA GÓMEZ A, TORRES RAMÍREZ C, MARTINEZ TORRES JL y cols.: Litiasis coraliforme: evolución de los cálculos residuales. Actas Urol Esp 1986; 10 (3): 191-196.

47. ZULUAGA GÓMEZ A.: Litiasis urinaria. En: Urología Vesalio. Coordinadores: Leiva Galvis O. y Resel Estevez L. ENE Ed., Madrid 1992: 323-348.

48. GASTÓN DE IRIARTE E, PERTUSA C, CÁRCAMO P, ORTÍN M, MARTÍNEZ-PIÑEIRO JA.: Tratamiento conservador de la litiasis urinaria: quimiolisis sistémica. En: Avances en Urología. Editado por Martínez-Piñeiro JA. Madrid (España), Reycosa ed. 1984: 199-216.

49. ROBERTSON WG, PEACOCK M, NORDIN BEC.: Calcium crystalluria in recurrent renal, stone formers. Lancet 1969; 2: 21-24.

50. ROBERTSON WG, NORDIN BEC.: Scientific foundations of urology. In: physico-chemical factors governing stone formation. Williams DI, Chisholm GD, eds. London: Heinemann 1976; 1: 254-267.

51. ETTINGER B.: Does hyperuricosuria play a role in calcium oxalate lithiasis?. J Urol 1989; 141: 738-741.

52. SABATINI S, ARRUDA JAL, KURTZMAN NA.: Trastornos del equilibrio acidobásico. Clin Med North Am 1978; 6: 1223-1255.

53. ALPERN RJ, STONE DK, RECTOR FC JR.: Renal acidification mechanisms. In: The Kidney, 4th ed. Edited by BM Brenner, FC Rector Jr. London, W.B. Saunders Co, 1991: 318-379.

54. BICHARA M, PAILLARD M, CORMAN B et al.: Volume expansion modulates $\mathrm{NaHCO}_{3}$ and $\mathrm{NaCl}$ transport in the proximal tubule and Henle's loop. Am J Physiol 1984; 247: F140-F150.

Dr. R.A. Medina López

Avda. Kansas City, 30 - Blq. 2, piso 1103

41007 Sevilla

(Trabajo recibido el 9 enero de 2003) 